Universidad de Lima

Facultad de Comuniación

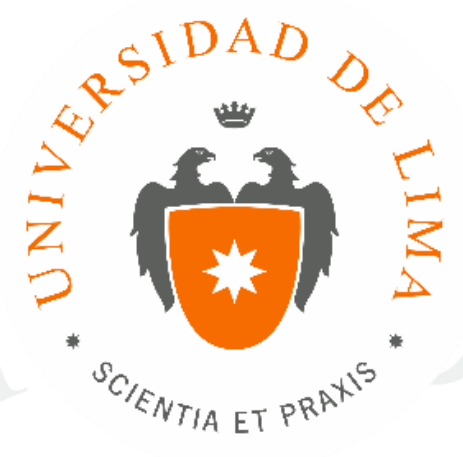

\title{
EL CONSUMIDOR DE TURISMO EN LÍNEA DE BAJO COSTO EN EL PERÚ. EL MUNDO DE LOS REVIEWS
}

Trabajo de investigación para optar la licenciatura de Comunicación

Marco Gervasi Pinto

Código 20100467

Asesor

Lilian Kanashiro

Lima - Perú

Mayo de 2016 


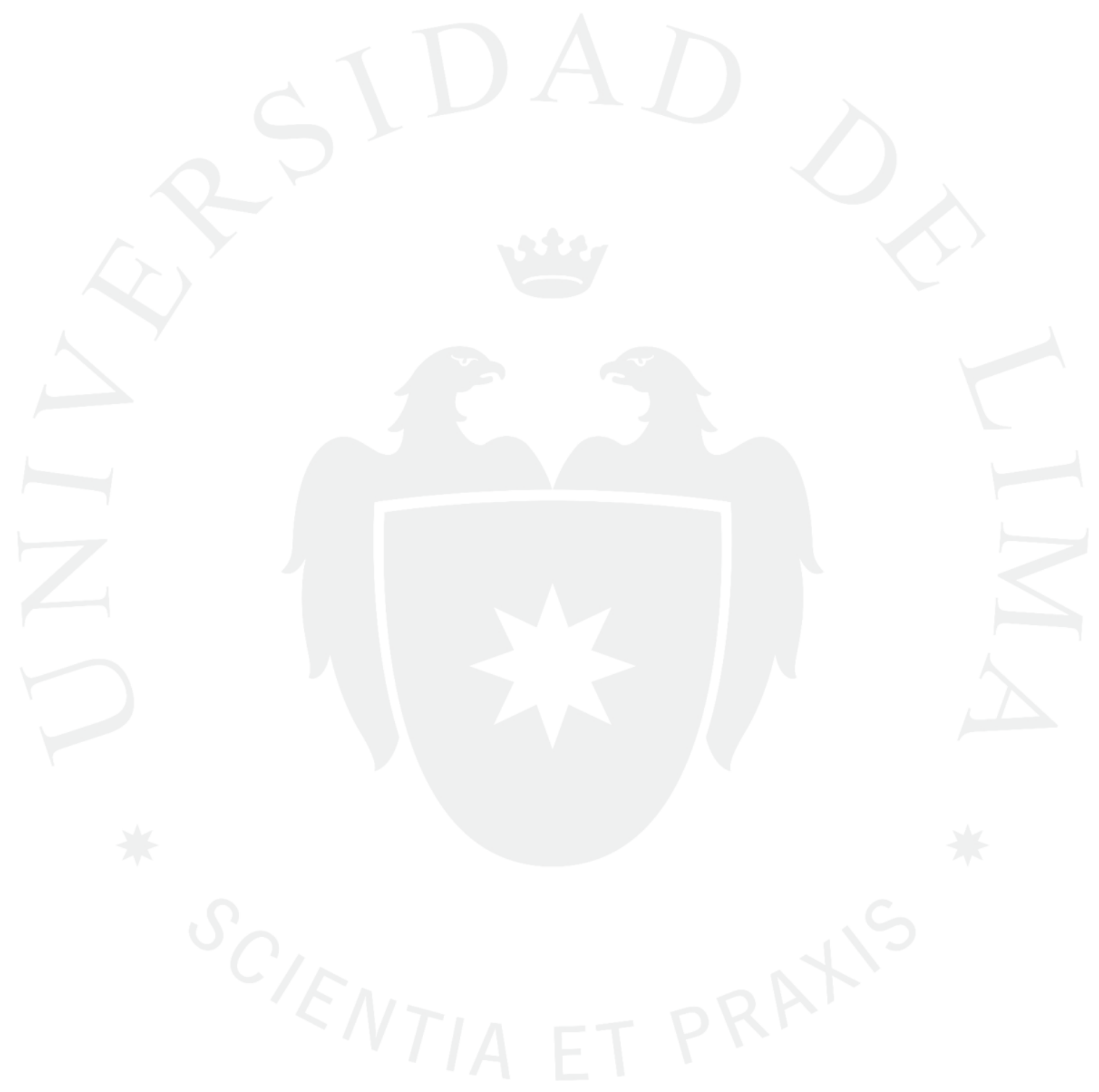




\section{EL CONSUMIDOR DE TURISMO EN LÍNEA DE BAJO COSTO EN EL PERÚ. EL MUNDO DE LOS REVIEWS}




\section{ÍNDICE}

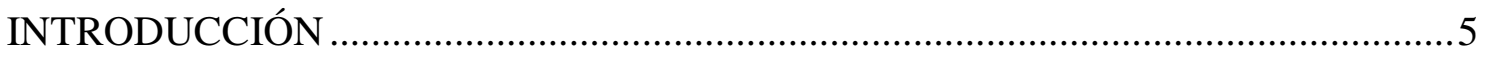

CAPÍTULO I. DEFINIENDO EL ENTORNO DIGITAL ..........................................

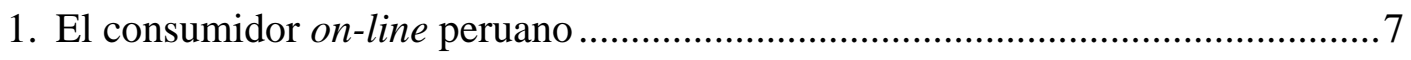

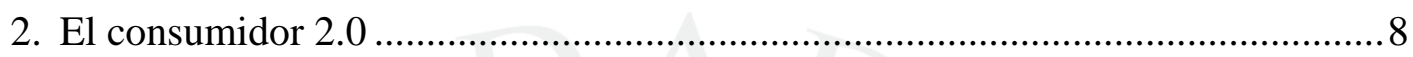

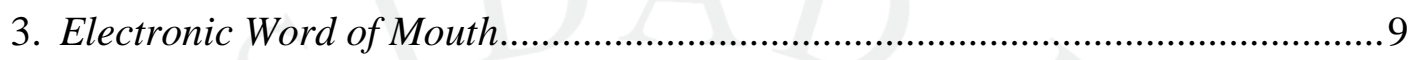

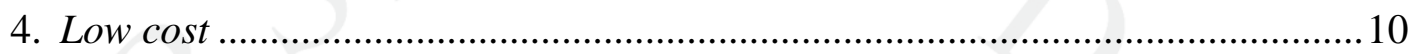

CAPÍTULO II. ESTADO DEL ARTE .................................................................... 11

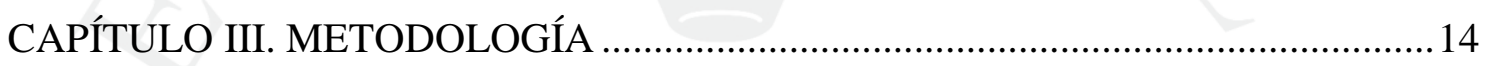

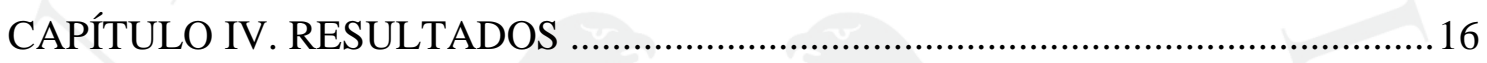

1. El turista peruano low cost viaja en grupos chicos, por periodos medios y prefiere ir a relajarse, pero quiere tocar muchos destinos ............................... 16

2. Los jóvenes peruanos utilizan las plataformas principalmente para hacer sus

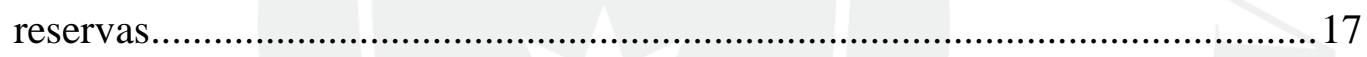

3. Los jóvenes peruanos utilizan las plataformas principalmente para hacer sus

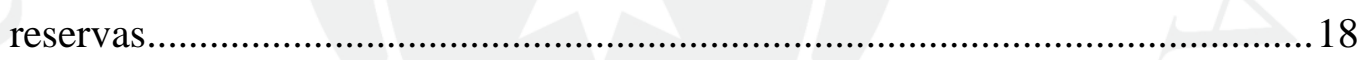

4. No hay mucho interés por la comida ......................................................... 19

5. Hay un gran interés por la experiencia del consumo ....................................20

6. El peruano prefiere comparar precios e irse por el más barato en la compra de

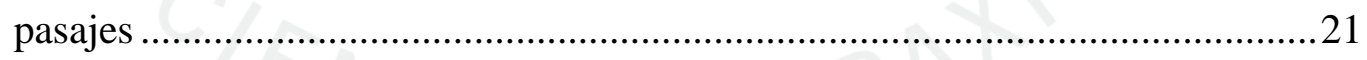

CAPÍTULO V. CONCLUSIONES Y RECOMENDACIONES ..................................22

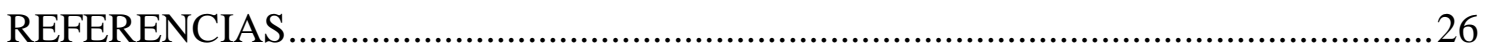




\section{INTRODUCCIÓN}

El turismo es uno de los puntos clave del desarrollo económico del Perú. El PBI Turístico representa el 3,9\% del total del país, El Consumo Turístico Interior representa $6,9 \%$ del gasto toal de la economía; además, el ingreso de divisisas por turismo asciende a US\$ 4,151 millones, significando un incremento de 47,5\% entre el 2011 y el 2015. (MINCETUR, 2016) En Perú, se cuenta con una de las principales maravillas del mundo y eso genera un flujo de cerca de 3 millones de visitantes anualmente (Xinhua 2013). En este contexto, es necesario saber que los consumidores ahora hacen todo on-line; según Canatur, el 70\% de peruanos consulta la oferta turística vía on-line y no viaja a menos de que sepan que el hotel o la experiencia que van a recibir va a ser placentera. A la luz de esto, se vuelve relevante saber cómo se comporta el consumidor peruano del turismo on-line.

Esta investigación busca describir algunos de los principales aspectos del comportamiento del consumidor on-line de turismo low-cost peruano, de 20 a 30 años de los NSE A y B, así como describir los insights de este nuevo consumidor que sirvan para generar un perfil utilizable en futuras investigaciones como una herramienta de información y referencia en el entorno digital.

Según los autores Zheng Xiang, Vincent P. Magnini y Daniel R. Fesenmaier, en general, la adopción de Internet ha alcanzado un nivel de saturación y algunos canales tradicionales, tales como agencias de viajes on-line, seguirán dominando la planificación de viajes (Xiang, Magnini \& Fesenmaier 2014). Sin embargo, con la llegada de las comunidades virtuales y las nuevas plataformas de turismo, ha surgido un nuevo turista, uno que no necesita de intermediarios para informarse, que gestiona sus propios destinos, contacta directamente con sus hoteles y maneja su propia experiencia a su medida; en otras palabras, es su propia agencia de viaje. En conclusión, luego de analizar las tendencias en las diferentes páginas web de turismo on-line y, tras una serie de grupos focales, se puede decir que hay un nuevo tipo de consumidor. Esto se puede apreciar con el enorme crecimiento de las principales páginas de turismo, como Tripadvisor.com o Hostelworld.com, y la participación de los turistas entre ellos; además del aumento anual del flujo de turismo internacional por los turistas de bajo presupuesto. También se puede ver en conductas nuevas con respecto al turismo, la 
búsqueda de albergues baratos, la lectura y escritura de comentarios sin esperar nada a cambio, la aparición de las comunidades de turismo y de valoraciones, como lo evidencian los ya mencionados grupos focales que se verán en la investigación. 


\section{CAPÍTULO I}

\section{DEFINIENDO EL ENTORNO DIGITAL}

Para esta investigación, al no haber una definición estándar o una teoría que englobe el tema, se van a tomar cuatro definiciones que van a ayudar a su mejor entendimiento, al dominio de términos y definiciones claves para el óptimo entendimiento de los resultados de la misma.

\section{El CONSUMIDOR ON-LINE PERUANO}

Según Sally Graham (2013), hay una pirámide de los hábitos de consumo online en la que, mientras se va subiendo cada escalón, se va pasando de una comunicación pasiva a una activa y, a la vez, se pasa de una relación abierta a una más íntima. Tenemos, en el escalón inferior, al consumo como base de la relación; luego, los comentarios, que ya requieren algo de proactividad; después está la conversación, que ya implica una comunicación lateral con otros consumidores; luego viene la colaboración; y finalmente la cocreación, en la cual se ubica solo el $10 \%$ de los consumidores. Según ella, los peruanos solo llegamos hasta el escalón de la conversación; no se llega a colaborar con las marcas y estas aún no lo permiten. Esto plantea que hay una falta de entendimiento y empatía por los consumidores.

Esta idea es muy importante, puesto que vemos que las marcas no están llegando a tener un contacto íntimo con los consumidores: se invierte más en campañas de branding que en generar experiencias inolvidables para los clientes más fieles. El consumidor on-line tiene que tener una experiencia agradable y única para que recomiende la página y vuelva.

Otra de las cosas que dice Graham es que el consumidor peruano no compra on-line a tiendas peruanas porque el mix del producto no es bueno. Las páginas no brindan un soporte lo suficientemente bueno y no se tiene una confianza en el pago y la entrega del producto.

Es entonces el consumidor peruano un ser de comunidades: se junta con los demás consumidores para compartir sus experiencias, para comparar sus valoraciones sobre un producto y así tomar mejores decisiones; sin embargo, no se suele gastar en 
productos por internet, lo que sí, es que se gasta en viajes. Pocas páginas — como la de LAN - se han ganado la confianza de los consumidores para un pago on-line. Y a esto se llegó a través de un boca a boca, pero digital. A esto se le llama Electronic Word-ofMouth o Boca-A-Boca Electrónico (e-WOM).

\section{EL CONSUMIDOR 2.0}

Un consumidor 2.0 es aquel que utiliza las bondades que ofrece Internet para hacer más rica su experiencia de compra y tiene una serie de ventajas que le brinda la gran variedad de herramientas que puede encontrar en la red. Cuando se habla de los consumidores 2.0, salta automáticamente el término "prosumidor".

Según se explica, un prosumidor es una persona que consume contenidos, pero que a la vez los produce; es decir, una persona que genera una comunicación multilateral: hacia arriba, con la empresa que produce algo; hacia el lado, con los demás consumidores 2.0; y hacia abajo, con las personas que aún no generan consumo (Naranjo, 2008). Sin embargo, para poder generar contenidos, nos damos cuenta de que los prosumidores son expertos en todo. Esto no significa que tengan el conocimiento absoluto de todo; pero, antes de hacer cualquier consumo, utilizan los recursos que tienen alrededor para informarse del tema, de manera que toman decisiones informadas: contrastan, averiguan, opinan, comparan, etc. (Duffill 2014). Lo que afirman Duffill y Naranjo refleja claramente la realidad de los turistas low cost. Estamos de acuerdo en que estos consumidores no solo reciben información de manera vertical; sino que producen comentarios, valoraciones, participan en blogs y redes sociales, además de no tomar una decisión de compra hasta estar sumamente informados acerca de sus destinos y alojamientos, relacionándose perfectamente con lo que nos dice Sally Graham.

A la vez, un consumidor 2.0 es uno que está siempre conectado (Duffill 2014), que está presente en el momento en el que se genera una comunicación, en el que se hace una publicidad, en el que se lanza un producto; pero también está presente cuando se genera una crítica negativa, cuando hay una falla con el producto, cuando hay un escándalo con la empresa. Es un consumidor que siempre está presente y no se le escapa nada.

Es un consumidor que elige un producto estando acorde con sus necesidades y deseos; no es necesariamente fiel a una marca, cambia de marca y de producto sin 
remordimiento. Utiliza las herramientas web para apoyar su búsqueda del producto que más se acomode a lo que él desea.

Se suele tratar de nativos digitales o inmigrantes digitales, personas que tienen un conocimiento amplio del uso de las computadoras, redes sociales y el Internet.

\section{ELECTRONIC WORD OF MOUTH}

Electronic Word-of-Mouth (e-WOM), según Robert Westbrook, alude a todas las comunicaciones informales dirigidas a los consumidores a través de la tecnología basada en Internet, relacionada con el uso o características de determinados bienes y servicios o sus vendedores. Esto incluye la comunicación entre los productores y los consumidores, así como las que existen entre los propios consumidores (Westbrook 1987). Cabe mencionar que no es lo mismo que las comunicaciones masivas, la publicidad o el marketing personalizado.

La recomendación de otra persona que ya ha tenido determinada experiencia me puede ayudar a saber si debería o no consumir el producto o servicio en cuestión. Para averiguar la manera en la que se efectúa el boca-a-boca en línea, para esta investigación se hizo una revisión de las principales páginas de compra de hoteles y de blogs de turismo y se descubrió que hay muchas formas de $e$-WOM: la principal es las valoraciones. Se suele tener, en una página, un área donde evaluar una experiencia sobre la estadía de los hospedajes o sobre la experiencia personal de cada uno relacionada a determinado sitio turístico, ya sea un alojamiento o un atractivo turístico; en estas, se suele evaluar del 1 al 10 o se suele hacer una escala, además de dejar un comentario que cuente a más detalle el porque de aquella evaluación. Además, hay una sección de comentarios en la que los interesados pueden compartir y conversar entre ellos. La otra forma en la que se suele hacer $e-W O M$ es a través de páginas de Facebook, blogs o foros.

La influencia del $e$-WOM ha demostrado ser positiva y a la vez negativa en los productos turísticos. Una valoración negativa puede tener un impacto enorme sobre la imagen de un hotel, ya que los visitantes insatisfechos van a propagar los comentarios poco halagadores relacionados con sus experiencias, y ya sabemos que los posibles consumidores los van a leer y posiblemente desanimarse. 
El impacto de las $e$-WOM es un factor tan grande que se puede ver que países como Nueva Zelanda: la elección de los destinos de los viajeros se basan en las recomendaciones que reciben a través de internet. Al igual que el consumo en restaurantes durante su viaje, son todos por el e-WOM (Litvin, Goldsmith, \& Pan 2008).

\section{LoW COST}

Low cost es un método de fijación de precios en el cual una empresa establece un precio comparativamente bajo con el fin de aumentar la demanda de su producto entre los consumidores, así como su posición competitiva en el mercado.

Una estrategia de precios bajos es un enfoque de marketing alternativo que una empresa puede utilizar como una alternativa a la diferenciación y centrar las estrategias de fijación de precios.

Esta estrategia tiende a ser más exitosa cuando el producto es bastante genérico y la producción de alto volumen es posible; o, en el caso de los servicios, si su sistematización o reducción de costos no quita el beneficio que pretende ofrecer el servicio (ya sea el dormir en un hotel o volar en un avión de un destino "A" a un destino "B").

Estos conceptos, nos ayudan a comprender mejor cómo es el consumidor peruano en línea, cómo se comunica, dónde lo hace, cómo ha evolucionado pasando a ser un consumidor 2.0 y cómo funciona la dinámica del boca-a-boca en línea. Teniendo una idea más clara de cómo es el consumo en línea, en especial del consumidor peruano, se da un paso más hacia poder descubrir cuál es el insight de estos consumidores. 


\section{CAPÍtULO II}

\section{ESTADO DEL ARTE}

El comportamiento del consumidor en el turismo on-line es un tema que se ha empezado a tratar desde el año 2003, año en que páginas como Hostelworld.com o Tripadvisor.com comenzaron a tomar relevancia por su utilidad para los jóvenes viajeros. Estas páginas trajeron un nuevo tipo de boca-a-boca, ya que en ellas podemos encontrar los llamados foros y valoraciones, cosa que hace que estos actúen de manera diferente frente a los productos que se le presentan en Internet.

Ipsos tiene dos investigaciones relevantes que pueden ser de ayuda para entender al consumidor on-line peruano. Primero, en su informe "Hábitos, usos y actitudes hacia Internet 2014", nos dice que: "Después de más de una década podemos decir que el internauta se ha vuelto un joven adulto estudiante y trabajador" (Ipsos Perú 2014a); pero más adelante explica que: “Quien realiza compras y/o ventas online es un joven adulto hombre del nivel socioeconómicos $\mathrm{A}, \mathrm{B}$ o $\mathrm{C}$ y que se encuentra trabajando en la actualidad" (Ipsos Perú 2014a).

Lamentablemente, también dice que aún son pocos los internautas que han incursionado en el mundo de las compras y ventas on-line: solo el $7 \%$ de la población ha consumido de esta manera. (Ipsos Perú 2014a). Y otro dato interesante es que, de la audiencia encuestada para esta investigación, de los que apostaron por la compra online, la mitad ha experimentado una compra en Mercado Libre, seguido de Facebook; lo que nos deja saber que son las principales páginas donde se da consumo on-line. Del total del consumo on-line, el $17 \%$ va hacia pasajes y solo el $1 \%$ hacia reservas de hotel. La información de este estudio dice que se trata de un mercado pequeño, pero esto no es necesariamente negativo; por más de que se trata de un mercado chico, es principalmente utilizado por los segmentos con mayor poder adquisitivo, haciéndolo un fuerte nicho a ser explotado.

Luego, en el informe "Perfil del internauta limeño 2014", explica que el internauta de NSE A/B es el "heavy user" de Internet, que lo hace de modo más portátil y quien más apuesta por el comercio electrónico (Ipsos Perú 2014b). 
En estas investigaciones, se puede ver que los internautas peruanos que consumen son pocos $\mathrm{y}$ esto se debe a que aún hay una gran desconfianza por los servicios on-line, por lo que se puede ver que el consumidor on-line es desconfiado y muy investigativo antes de realizar cualquier consumo.

En 2009, se publica una investigación acerca del impacto de los reviews de los hoteles en la consideración de los consumidores para hospedarse ahí: "Tried and tested: The impact of online hotel reviews on consumer consideration"; el cual fue un estudio cuantitativo y cualitatativo donde se cruzaron muchas variables y estadísticas. Para los hoteles que tenían poca notoriedad, como los hoteles mochileros o pequeños, un review era muy impactante; mientras que hoteles grandes — como la cadena Hilton- no se veían afectados por los reviews (Vermeulen \& Seegers 2009). La investigación termina concluyendo que los reviews hacen que cambie la percepción de un hotel, hasta puede hacer que cambie la actitud de un mismo hotel hacia sus clientes. Cruzando esto con lo dicho por Ipsos, podemos ver que, si el consumidor peruano es tan desconfiado, puede verse muy afectado por una valoración negativa de un cliente insatisfecho, por lo que deberían esforzarse más en mantener felices a sus consumidores.

Zheng Xiang y Ulrike Gretzel condujeron una investigación acerca del rol de las social media en la búsqueda de información para viajes. Según ellos, podemos definir al turismo on-line como un dominio donde se recopila toda la información acerca de lo relacionado a un viaje: links, información, precios, ofertas, valoraciones, etc. (Xiang \& Gretzel 2010). Para comprobar su rol en el turismo on-line, gracias a un par de investigaciones cuantitativas, primero analizaron la cantidad de páginas de búsqueda de turismo on-line existentes: encontraron que habían 10 383; sin embargo, solo el $6 \%$ de las más visitadas se llevaban más del 50\% del flujo de consumidores. Luego se analizó los diferentes tipos de redes sociales que se podían utilizar, encontrando blogs, redes sociales, media, comunidades virtuales y las valoraciones o reviews. Vieron que más del $40 \%$ de las personas utilizaba las comunidades virtuales, cosa que llamó su atención. En el ranking de las veinte páginas más utilizadas para el turismo on-line, Tripadvisor.com se encontraba primera, la cual se basa en una comunidad virtual que genera puntuaciones y recomendaciones a través de los otros usuarios.

Luego se han hecho un análisis de cómo se comportan los turistas a la hora de reservar en línea. Tres autores provenientes de China nos afirman que la reserva de 
viajes on-line es comprar en línea con antelación y luego experimentar el servicio cara a cara. Para las empresas de viaje, es imprescindible proporcionar reservas on-line para mejorar la eficiencia del servicio, la calidad y obtener ventajas competitivas (Peng, Xu \& Chen 2013). El artículo propone una nueva agenda de investigación para los comportamientos turísticos en línea de reserva basada en revisión de la literatura de las tres etapas del proceso de reserva; resume los resultados en la búsqueda de información en línea, los determinantes de la línea amarilla y la lealtad de reserva en línea (Peng, $\mathrm{Xu}, \&$ Chen 2013).

A la vez, podemos ver cómo es el comportamiento de compra de los consumidores on-line. La página Principiar.com lanzó un perfil del consumidor on-line para el año 2014, en el cual se exponen puntos sobre cómo y dónde compra, qué tan seguido compra lo hace, qué compra y sus formas de pago. En este perfil, encontramos que suelen comprar en casa y pagar con tarjeta; además, la compra puede llegar a ser quincenal y el uso de herramientas como el smartphone o las tablets han incrementado el consumo on-line. Lo interesante de este perfil es que dice que en el sector en el que más se gasta es el del turismo y transporte (Principiar 2014). 


\section{CAPÍTULO III}

\section{METODOLOGÍA}

Esta investigación tiene como fin realizar un perfil del consumidor de turismo on-line low-cost en el Perú; para lo cual se hizo una investigación de tipo cualitativa, en la que se pudo observar y analizar la forma en la que estos se comportan y cómo son.

Se utilizaron los principales buscadores de alojamiento en busca de comentarios de consumidores peruanos para motivos de la investigación. Como las páginas que más afluencia de turistas tienen son Tripadvisor.com, Hostelworld.com y Bookings.com, estas fueron las utilizadas.

Para empezar, se empezó buscando los principales destinos turísticos del extranjero: Francia, Estados Unidos, España, Turquía e Italia (OMS 2014), etc. Dentro de la gran lista de comentarios de cada hotel o albergue, se encontraron reducidos comentarios de turistas peruanos. Es probable que se deba a que hay una baja afluencia de turistas de bajo costo al extranjero, a la falta de un dispositivo y los problemas de conectividad que trae consigo un viaje, o a que el turista peruano no tenga una cultura de comentarios evaluando los hoteles donde se ha hospedado. Suele dejarse comentarios cuando ha pasado algo fuera de lo ordinario o se está sumamente complacido con el trato y la experiencia; es decir, un comentario es como un premio o un castigo a lo que se sale de lo común.

Se usó la etnografía virtual (o netnografía). Esta técnica, al igual que la etnografía clásica, utiliza técnicas de investigación tales como las entrevistas, la observación, los grupos de discusión, etc., solo con la particularidad de hacerlo a través de Internet y gracias a los nuevos dispositivos electrónicos (smartphones, tablets, portátiles, etc.). Algunos lugares donde se puede realizar son: foros, chats, redes sociales, Messenger, correo electrónico, entornos virtuales, juegos on-line, etc. (Hine 2004).

Así, se realizó un análisis de contenidos de los comentarios, valoraciones e interacciones de los usuarios de páginas como Tripadvisor.com, HostelWorld.com y Booking.com. Para esto se elaboró una base de datos de los comentarios recopilados 
realizados por turistas peruanos y, a través de esta, se separó en temas en ejes comunes y mediante el análisis de estos se procesó el insight de los consumidores.

Además, se realizaron cuatro grupos focales en salas especializadas en la Universidad de Lima, con seis turistas peruanos por grupo. Dos se realizaron a jóvenes adultos de 20 a 25 años (desde ahora llamados jóvenes para facilitar la lectura de los resultados) y, los otros dos, a jóvenes adultos de 26 a 30 años (ahora llamados adultos). Se separaron por edad debido a que, como nos dicen David Morgan y Richard Krueger, para cuatro focus group, debemos evitar hacer más de una división por segmentación:

For example, if you're doing groups with high school students and need to separate them into boys and girls, you'll need at least two groups with boys and two with girls, for a total of four groups. The more splits you make, the more complicated the planning, so only split when necessary (Morgan \& Krueger 1997).

La población investigada estuvo compuesta por consumidores de turismo online low cost, de 20 a 30 años de edad, peruanos de los NSE A y B. Se eligió a esta población por diferentes motivos. Primero, porque a partir de los 20 años ya se ha alcanzado, en la mayoría de los casos, un cierto nivel de madurez e independencia para poder viajar sin compañía de padres o guardianes; luego, en este rango (los jóvenes adultos) se tiene una edad en la que no es vital para un viaje tener todas las comodidades de un hotel que no caiga dentro de la categoría del low cost; finalmente, pertenecen a los niveles socioeconómicos A y B porque, por más de que se trate de turismo low cost, el costo de un viaje requiere un determinado poder adquisitivo. 


\section{CAPÍTULO IV}

\section{RESULTADOS}

\section{El turista peruano low cost Viaja en grupos Chicos, por periodos MEDIOS Y PREFIERE IR A RELAJARSE, PERO QUIERE TOCAR MUCHOS DESTINOS}

Para los jóvenes de 20 a 25 años, encontramos que la mayoría prefiere viajar en grupos de cuatro personas como máximo, por motivos de coordinaciones y comodidad: "Yo he viajado normalmente en grupos de 4, es más fácil, si el grupo es muy grande se pueden perder, separarse en pequeños grupos y se hace desorden" (Jacky, 22 años); “[...] yo también estoy de acuerdo, 3 o 4 máximo porque un día unos pueden querer hacer una cosa y otros otra y es difícil ponerse de acuerdo" (Daniela, 24 años). Mientras que los adultos de 25 a 30 afirmaron que preferían viajar con una pareja: “[...] yo solo viajo con mi enamorado [...]” (Maria José, 26 años); “[...] yo por lo general [viajo] en pareja" (Julio, 29 años); "Yo siempre viajo con mi esposo" (Romina, 27 años).

En cuanto a la duración de sus viajes, los jóvenes dijeron que el tiempo promedio era de dos semanas: "[...] de preferencia viajes largos, por ejemplo yo hice un viaje largo de treinta días y a los últimos días ya tenía migraña, no podía más, el ritmo del turista es súper acelerado. La duración perfecta es dos semanas o tres y cuatro destinos máximo" (Alessandra, 23 años); “Obviamente un turista de nuestro tipo va a estar más acelerado que un turista normal, nunca tienes un itinerario hecho al 100\%, es casi ir a la deriva. El tiempo máximo yo creo que deben ser dos semanas y ya si quieres rajarte tres" (Maria Fé, 21 años). Por otro lado, los adultos concordaron en cuatro días o un fin de semana largo: "[...] una semana máximo" (Daniela, 26 años); “[...] un fin de semana o cinco días" (Manuel, 26 años).

Además, durante los viajes los jóvenes suelen visitar de tres a cuatro destinos; mientras que los mayores preferían quedarse en un solo destino, pero conocerlo a profundidad: "Solo me quedo en un sitio yo" (Manuel, 26 años); “[...] yo también solo prefiero un destino" (Julio, 29 años).

El destino favorito es la playa. Para los adultos, es mejor ir a relajarse solo en la playa: “[...] solo playa, yo quiero relajo total, no me importa nada de la cultura ni 
nada [risas]" (María José, 27 años); mientras que los más jóvenes tienen deseos de conocer la cultura, la ciudad, los monumentos y la historia: "Yo preferiría playa pero me encanta conocer monumentos" (Vera, 20 años); "Me gusta el vivencial lo turista, me gusta ir donde van los lugareños, en la experiencia de la gente local meterme en la experiencia de estar en el lugar" (Paloma, 22 años).

Sorprendentemente, gran parte de los jóvenes prefieren dormir bien para poder caminar y hacer turismo con más energía que salir a conocer la vida nocturna; mientras que los más adultos admiten que no se perderían una noche en la ciudad sin salir: "[...] es bonito salir, pero si al día siguiente necesitas hacer algo muy cansado es mejor echarte a descansar" (Paola, 23 años).

\section{LOS JÓVENES PERUANOS UTILIZAN LAS PLATAFORMAS PRINCIPALMENTE PARA HACER SUS RESERVAS}

Mientras que los menores afirmaron utilizar plataformas más recurridas como Tripadvisor.com, los del grupo de los mayores afirmaron utilizar buscadores generales como Despegar.com o las páginas de las aerolíneas para encontrar ofertas. Ambos grupos no descartaron el uso del boca-a-boca tradicional para la búsqueda de información de viajes: “[...] más que nada Tripadvisor” (Paloma, 22 años); "Prefiero Hostelworld, pero antes las recomendaciones de las personas y lo que has escuchado siempre de ese país, si lo has visto en una película o una serie" (Maria Fé, 21 años).

El peruano va más allá de $e$-WOM y utiliza también el boca-a-boca tradicional, recurriendo a amigos de la zona o a otras personas que hayan viajado para conseguir información acerca del lugar que va a visitar.

Dentro de las plataformas, los jóvenes no dejan de revisar los comentarios; mientras que los adultos no suelen fijarse mucho en eso y suelen fijarse más en fotografías o videos acerca de lo que tienen interés en comprar: "Sí, yo me revisaba toditos los comentarios, porque si no chequeabas bien podías acabar yendo a un sitio lleno de cucarachas o cochino" (Andrea, 22 años); “[...] yo no revisaba para nada, si me salía el porcentaje lo revisaba, pero no leía los comentarios uno por uno" (Nicolás, 26 años). 


\section{LOS VIAJEROS PERUANOS SON SU PROPIA AGENCIA DE VIAJE}

Para realizar sus compras en alojamientos, los jóvenes prefieren hacerlas en casa, tener una buena silla o sillón, internet y tranquilidad; mientras que los mayores no toman tanto en cuenta dónde hacen sus reservas, haciéndolo normalmente en la oficina: "Yo soy muy miedosa prefiero en casa" (Vera, 20) "En mi casa, en la oficina he buscado” (Romina, 27 años); “[...] yo en la oficina” (María José, 27 años).

Para alojamiento, los mayores afirmaron utilizar páginas como la sección de hostales de Despegar.com; mientras que los jóvenes utilizan diferentes páginas, como Booking.com, Hostelworld.com, Tripadvisor.com y cuanta encuentren.

Los adultos dijeron que como máximo revisan una o dos páginas; mientras que los jóvenes afirmaron revisar múltiples páginas por múltiples horas, hasta estar completamente satisfechos con el lugar que van a elegir: "[...] varias opciones 3, 4 o 5" (Daniela, 23 años); “[...] miles de ventanas” (Jacky, 21 años).

En cuanto a la influencia de los comentarios en su decisión de compra, los jóvenes dijeron que era sumamente importante leer lo que los demás tienen que decir acerca del sitio antes de tomarlo; mientras que los mayores dijeron que podía ser cosa de una persona que estuviera insatisfecha y preferían ver por ellos mismos.

Para los jóvenes, lo más importante es la ubicación a la hora de elegir un alojamiento; mientras que para los adultos es más importante revisar las fotos que tienen que ofrecer un lugar y, si les parece agradable, lo toman. La gran mayoría de los comentarios encontrados en las plataformas on-line hacían mención de la ubicación de los hoteles donde se quedaban los turistas, si estaban cerca o no de algún punto estratégico, atracción turística o el centro de la ciudad: “[...] yo pongo ubicación sobre precio, porque en moverte por la ciudad dentro de una ciudad grande puede resultar caro y acabas gastando más" (Alessandra, 23 años); “[...] yo primero reviso las fotos del hotel y si no me gusta, no" (Julio Gabriel, 26 años); "9,2 La ubicación (negativo), debido a que está un poco lejos del centro, pero la estación de tranvía está a 100 mts" (comentario en Tripadvisor.com).

Los viajeros buscan una buena conexión a Internet. Durante un viaje, luego de caminar muchas horas y tomar fotos por las calles de la ciudad visitada, vuelven a sus hospedajes a hablar con sus amigos y familia, compartir sus fotos y leer sus correos electrónicos. Para esto, es necesario tener una buena conexión a Internet y brindar un 
buen servicio wi-fi. Se encontraron comentarios acerca del wi-fi en las plataformas, además de valoraciones positivas de este servicio: "8,3 en las habitaciones no llega la señal del wifi”" (comentario en Booking.com).

Los jóvenes admitieron no tener problemas compartiendo su habitación con gente que no conocían con tal de que sea más barato; mientras que los adultos afirmaron que sí pagarían una diferencia de precio para tener un cuarto privado.

Al peruano le preocupa su seguridad antes que otras cosas. A la hora de elegir un alojamiento para los turistas peruanos, la seguridad se convierte en el segundo tema más importante. Se encontró algunos comentarios reflejando esto, principalmente haciendo referencia a situaciones en las que su seguridad se había visto afectada de alguna manera:

Dejé mis zapatillas y balerinas al lado de mi cama, cuando volví de pasear no las encontré. Pregunté en recepción y me dijeron que averiguarían. Cuando regresé me las entregaron sin darme ninguna explicación de quién ni por qué las habían tomado (comentario en Booking.com).

Muy bueno, solo una observación con los lockers. Todo muy bueno, buen servicio, siempre tienen ganas de atenderte bien y divertirte... Solo una observación: En las dos habitaciones que estuve justo me asignaron lockers cuyos cerrojos estaban malogrados, tuve que llamar a recepción para que un cerrajero lo solucione. Mientras tanto, no tuve dónde guardar mis cosas. Además, quise comprar candados para los lockers pero ya se les habían acabado. Eso fue lo único que pudo mejorar en mi estadía (comentario en Booking.com).

\section{NO HAY MUCHO INTERÉS POR LA COMIDA}

Cuando viajan, los jóvenes aseguraron no tener mucho interés por la comida; la mayoría compra ingredientes no perecibles y prepara algo para comer en la calle de manera rápida y barata. A su vez, se dijo que una de las principales fuentes de su alimentación durante sus viajes son las principales cadenas de fast food: "[...] nosotros armábamos sándwiches y los llevábamos en nuestras carteras para el almuerzo" (Flor, 25 años); “[...] en el hotel o un restaurante cerca o hay veces que hasta no como para seguir avanzando" (María José, 26 años). 
Los jóvenes eligen el lugar al que van a comer mientras caminan por la ciudad $\mathrm{y}$ van haciendo turismo, escogiendo uno que tenga una apariencia de acuerdo a lo que ellos buscan; mientras que los adultos suelen revisar en el hotel donde se quedan o pedir una recomendación en la calle de algún buen lugar donde puedan comer.

Todos los encuestados concuerdan en tener interés por probar la comida local, afirmando que es parte importante de llegar a conocer un lugar, por lo menos un platillo típico: “[...] es algo súper importante, ya sea algo dulce o salado es parte básica de la experiencia” (Tsiulang, 26 años); “[...] por lo menos alguna cosita he tenido que probar por ahí" (Andrea, 23 años).

\section{HAY UN GRAN INTERÉS POR LA EXPERIENCIA DEL CONSUMO}

En las plataformas on-line, podemos ver que el factor más mencionado dentro de los comentarios encontrados fue el ambiente del restaurante, la decoración, el espacio, el orden, la limpieza, la música, el personal y también algunas cosas más intangibles, como la vibra y el ánimo: "Muy buen lugar, muy acogedor, pequeño. Cuando he ido siempre ha habido sitios disponibles. Buscan innovar con los platos. Muy recomendable. La atención es buena, y los precios también!" (comentario en Tripadvisor.com). Otro comentario menciona:

Muy agradable (5 de 5) El ambiente es muy acogedor y la estructura original de sillar tipo bóveda es un plus por que lo convierte en un lugar digno de compartir una buena cena. La cocina o sea sus platos son muy bien elaborados como las carnes de vacuno, cerdo (ya no hay de avestruz) en piedra traída se Suiza, son muy ricos ya que uno puede manejar el grado de cocción. Además el manejo de la quinua cosida o sea cocinada es también un plus. He ido muchas veces así como años nuevos. Buenos tragos, buen vino y los dueños grandes anfitriones. Salud por todos. Hugo (comentario de Tripadvisor.com).

Otro factor que consideran los peruanos en sus comentarios es acerca de la calidad de la comida. Muchos de los comentarios encontrados en los restaurantes peruanos eran acerca de la calidad de la comida o del plato favorito del consumidor en particular: "Rico y más (5 de 5) Soy fan de los postres, y estos me dejaron con ganas de más. NO es porque se sirva poquito (que tal vez más grande no estaría mal) sino porque el sabor es excepcional. Muy recomendado" (comentario en Tripadvisor.com). 
Vemos que parte de la experiencia de un restaurante es la atención. Muchos de los comentarios tienen que ver con el trato que se les brinda a los clientes a la hora de llegar al restaurante: si se les atendió bien o mal.

\section{El PERUANO PREFIERE COMPARAR PRECIOS E IRSE POR EL MÁS BARATO EN LA COMPRA DE PASAJES}

Los jóvenes afirmaron comprar sus pasajes en buscadores de viajes en general, como Despegar.com, Skyscanner.com u otro que encuentren en buscadores como Google o Yahoo!; mientras que los adultos prefieren utilizar una página de una aerolínea, como Lan.com o AmericanAirlines.com: "Yo usaba una página que te chequeaba todos los precios de todos los vuelos y ahí comparaba” (Alessandra, 23 años); “[...] las páginas de LAN así” (Tsiulang, 26 años).

A la hora de comprar sus pasajes, fue un común denominador encontrar que todos priorizaban el precio más allá de la comodidad, los beneficios o el horario de los vuelos: "[...] yo chequeo el precio, solamente" (Daniela, 26 años); "Precio de todas maneras" (Andrea, 23 años). 


\section{CAPÍtULO V}

\section{CONCLUSIONES Y RECOMENDACIONES}

Tras la investigación realizada, creemos que se logró tener un perfil mucho más claro del consumidor de turismo on-line low cost en el Perú, mostrando algunas de sus peculiaridades y generando algunos análisis de su comportamiento.

Zheng Xiang y Ulrike Gretzel comentan que el 6\% de las páginas se lleva el $60 \%$ del flujo de los turistas. Luego de ver los resultados encontrados en los focus groups y de revisar las principales páginas de turismo, es seguro decir que esto es correcto y también aplica a los turistas low cost peruanos. Ello se puede deber a que, como se dijo en los grupos, para comenzar a encontrar plataformas a la cuales colgarse, los internautas viajeros ingresan primero a Google y hacen una búsqueda, pudiendo ser que los primeros resultados sean los que más clics reciben, poniendo en ventaja a las grandes páginas como Tripadvisor.com o Booking.com. Otro factor que juega un rol importante en el flujo de las páginas es el de las recomendaciones boca-a-boca, las cuales, como vimos, los viajeros peruanos suelen utilizar para complementar sus búsquedas de información.

Los comentarios claramente tienen un efecto sobre el comprador de turismo on-line peruano. Vemos que los turistas peruanos sí revisan siempre los comentarios que dejan el resto de los turistas acerca de los hoteles y restaurantes. Esto comprueba lo que dicen Vermeulen y Seegers acerca de los resultados de los focus groups realizados: al leer reviews negativos, los turistas peruanos cambian su actitud hacia lo que están interesados en comprar. Como se mencionó en los resultados, los jóvenes dijeron que era sumamente importante leer lo que los demás tienen que decir acerca del sitio antes de tomarlo; además, están dispuestos a pasar muchas horas buscando algo que se acomode a lo que ellos necesitan, abriendo varias páginas y comparando muchas opciones antes de tomar la adecuada. Sin embargo, nos encontramos con que, por más que los viajeros peruanos se dediquen a leer los comentarios y valoraciones, es común que ellos mismos no dejen una para los demás viajeros.

Sally Graham nos habla de la pirámide de los hábitos de consumo on-line (mientras se va subiendo cada escalón, se va pasando de una comunicación pasiva a una 
activa) y dice que el consumidor peruano llega hasta el escalón de la conversación, donde no se llega a colaborar con las marcas, ya que estas aún no lo permiten. Ella plantea que hay una falta de entendimiento y empatía por los consumidores. Lamentablemente, podemos ver que, en el caso del turismo en línea, no se llega ni a ese escalón; como ya mencionamos, los consumidores solamente llegan a leer los comentarios de los hoteles y restaurantes y la gran mayoría suele evitar comentar porque les da pereza y no consideran que es una buena inversión de su tiempo.

Se menciona que hay un gran interés por la experiencia del consumo, lo que se relaciona mucho con la definición base del marketing turístico, el cual no se dedica a vender bienes ni servicios, sino experiencias. Basta con brindarle una buena experiencia a un viajero para que, satisfecho, haga uso del $e$-WOM; ya que se sabe que para que un turista peruano deje un comentario tiene que ser una experiencia que rompa totalmente con el umbral de experiencias al que está acostumbrado (ya sea buena o mala la experiencia). Lo particular del turista peruano es que no se queda solo en el boca-a-boca electrónico, sino que también aplica el tradicional para complementar su recomendación.

Los viajeros del tipo low cost peruanos tienen la particularidad de ser muy funcionales: aprovechan al máximo su estadía en los lugares que visitan, se informan de las mejores rutas, se alojan cerca de las principales atracciones o líneas de transporte, comen rápido y barato, evitan los grandes grupos para viajar cómodamente y no temen utilizar el transporte público para llegar a sus destinos. Esto no tiene mucho que ver con el estilo de vida que se suele llevar en nuestra ciudad, especialmente para los NSE investigados, donde normalmente no se come en sitios baratos, duermen en cuartos compartidos y mucho menos se utiliza el transporte público. Esto demuestra que el turista peruano cambia su mentalidad cuando está viajando: son personas versátiles que están dispuestos a dejar de lado sus lujos para poder conocer el mundo con el presupuesto ajustado.

No hay un interés grande por la comida a la hora de viajar. Esto puede tener que ver con el cambio de mentalidad de los turistas a la hora de encontrarse viajando, pero también puede ser un asunto de patriotismo. Se sabe que la comida peruana es la mejor del mundo; entonces, es común que el viajero peruano tenga un paladar muy exquisito y a lo mejor se sienta impresionado con los platos que se le puedan presentar. 
Por eso, puede ser que no se llegue a romper con el umbral de experiencias al que está acostumbrado a vivir el peruano, con el buen trato y gastronomía de su propio país.

También se puede decir que los turistas peruanos hacen una minuciosa investigación antes de tomar su destino. No suele importarles pasar horas buscando el mejor hotel para su viaje o buscando los mejores destinos para poder visitar. Son viajeros organizados que planean mucho y se informan. Como se explica en el marco referencial, se trata de un consumidor 2.0 que "sabe de todo" y utiliza plenamente las herramientas que le brinda Internet para sacarle el máximo provecho al tiempo que va a pasar viajando.

Se plantea la duda si acaso el turista on-line está saturado por la Internet y termina recurriendo a las agencias de viaje para armar su itinerario y elegir sus destinos. Zheng Xiang, Vincent Magnini y Daniel Fesenmaier plantean esta idea como algo que refleja el exceso de la exposición al Internet que tienen los usuarios. Lamentablemente, esto queda totalmente descartado cuando vemos que este particular turista utiliza sus propios medios para armar un viaje hecho a su medida y con los destinos que él desea. Desde la decisión de adquirir su pasaje por sus propios medios, él mismo se informa y averigua a través de las redes sociales y las plataformas de turismo. La decisión de la compra de pasajes implica una fuerte investigación hecha por ellos mismos, desde la elección de la aerolínea hasta la comparación de precios que conlleva ser un turista low cost. Luego, la elección de un hotel (que implica revisar diversas páginas), la ubicación de los hoteles, el precio de cada habitación, la lectura de los comentarios de todos los demás turistas, etc. Vemos que él mismo gestiona su viaje, siendo su propia agencia, asesorándose de los recursos que tiene a la mano con las plataformas de turismo y las redes sociales.

Para futuras investigaciones, se recomienda complementar todo lo dicho con una investigación desde el otro lado del negocio del turismo low cost. Se podría hacer una investigación acerca de cómo tiene que ser el comportamiento de un negocio turístico para amoldarse a la nuevas exigencias de los viajeros de bajo precio.

Este artículo puede servir como herramienta para seguir los lineamientos presentados en los resultados acerca del comportamiento del consumidor y para mejorar el servicio brindado para los turistas del segmento. Incluso, se presta para generar un 
perfil del tipo de consumidor que puede servir para la segmentación de marketing y para futuras investigaciones en el mercado. 


\section{REFERENCIAS}

Ali, Rafat (2014). The Top Online Travel Booking Sites for January 2014. Recuperado de: http://skift.com/2014/02/24/the-top-online-travel-booking-sites-for-january2014/

Beerli, Asunción \& Josefa Martín (2002). El proceso de formación de la imagen de los destinos: una revisión teórica. Estudios turísticos, 154, 5-32.

Duffill, Mariana (2014). ¿Cómo es el Consumidor 2.0? Recuperado de: http://www.creatiburon.com/como-es-el-consumidor-2-0/

Graham, Sally (2013). El consumidor digital peruano: necesidades y espectativas (conferencia dada en la UCAL). Recuperado de: http://es.slideshare.net/sallygrahams/consumidor-digital-peruano-2013

Hine, Christine (2004). Etnografía virtual. Barcelona: UOC.

Ipsos Perú (2014a). Hábitos, usos y actitudes hacia Internet 2014. Lima: Ipsos.

Ipsos Perú (2014b). Perfil del internauta limeño. Lima: Ipsos.

Litvin, Stephen, Ronald Goldsmith \& Bing Pan (2008). Electronic word-of-mouth in hospitality and tourism management. Tourism Management, 29(3), 458-468.

Morgan, David \& Richard Krueger (1997). The Focus Group Kit (volumen 3). Louisville, Estados Unidos: SAGE Publications.

Naranjo, Daniel (2008). El consumidor 2.0. Colombia: Mkthink.

Organización Mundial de Turismo (OMT) (2014). Panorama OMS del Turismo Mundial. Madrid: OMT.

Peng, Hongxia, Xianhao Xu \& Wen Chen (2013). Tourist behaviours in online booking: A new research agenda. Communications in Information Science and Management Engineering, 3(6), 280-285.

Principiar (2014). Perfil del consumidor online para el 2014. Recuperado de: http://www.principiar.com/blog/perfil-del-consumidor-online-para-el-2014/

Ministerio de Comercio Exterior y Turismo del Perú. (2016). Medición Económica del Turismo. Lima, Perú: MINCETUR. 
Vermeulen, Ivar \& Daphne Seegers (2009). Tried and tested: The impact of online hotel reviews on consumer consideration. Tourism Management, 30(1), 123-127.

Westbrook, Robert (1987). Product/consumption-based affective responses and postpurchase. Journal of Marketing Research, 24(3), 258-270.

Xiang, Zheng \& Ulrike Gretzel (2010). Role of social media in online travel information search. Tourism Management, 31(2), 179-188.

Xiang, Zheng, Vincent P. Magnini \& Daniel R. Fesenmaier (2015). Information technology and consumer behavior in travel and tourism: Insights from travel planning using the internet. Journal of Retailing and Consumer Services, 22, 244-249.

Xinhua (2013). Transacciones online en el sector turismo de Perú superan los US\$1.200M al año. Recuperado de America Economía: http://www.americaeconomia.com/node/102397 